\title{
Feasibility Study on the Use of the Seeding Growth Technique in Producing a Highly Stable Gold Nanoparticle Colloidal System
}

\author{
Kim Han Tan, ${ }^{1,2}$ Hairul Anuar Tajuddin, ${ }^{3}$ and Mohd Rafie Johan ${ }^{1,2}$ \\ ${ }^{1}$ Nanomaterials Engineering Research Group, Advanced Materials Research Laboratory, Department of Mechanical Engineering, \\ Faculty of Engineering, University of Malaya, 50603 Kuala Lumpur, Malaysia \\ ${ }^{2}$ Materials Engineering Unit, Faculty of Engineering, University of Malaya, 50603 Kuala Lumpur, Malaysia \\ ${ }^{3}$ Organic Research Laboratory, Blue Level, Department of Chemistry, Faculty of Science, University of Malaya, \\ 50603 Kuala Lumpur, Malaysia \\ Correspondence should be addressed to Kim Han Tan; kimhan8419@gmail.com
}

Received 16 February 2015; Revised 30 March 2015; Accepted 9 April 2015

Academic Editor: Lavinia Balan

Copyright (c) 2015 Kim Han Tan et al. This is an open access article distributed under the Creative Commons Attribution License, which permits unrestricted use, distribution, and reproduction in any medium, provided the original work is properly cited.

Stable colloidal gold nanoparticles (Au NPs) are synthesized successfully using a seeding growth technique. The size of the nanoparticles is determined using transmission electron microscopy (TEM), and it is observed that the size of the nanoparticles ranges from 7 to $30 \mathrm{~nm}$. The TEM images and optical absorption spectra of the Au NPs reveal that the suspension is well dispersed and consistent with the particle size. The feasibility of the seeding growth technique is investigated using Turbiscan Classic MA 2000 screening stability tester. Based on the peak thickness kinetics and mean value kinetics, the backscattered light profiles indicate that the suspension is highly stable without particle sedimentation as well as negligible agglomeration. In addition, the Au NPs are proven to remain stable over a period of 2 months. Particle sedimentation eventually occurs due to the weight of nanoparticles. It is concluded that the seeding growth technique is feasible in synthesizing stable Au NPs. Controlling the stability, size and shape of Au NPs are technologically important because of the strong correlation between these parameters and the optical, electrical, and catalytic properties of the nanoparticles.

\section{Introduction}

Noble metal nanoparticles (NPs) are important candidates for photonics, plasmonics, and metamaterials (MMs) applications [1]. They have attracted considerable interest among researchers over the years because of their unique properties, that is, localized surface plasmon resonance (LSPR). LSPR refers to the resonant oscillation of the free electrons of the NPs in the presence of light. The LSPR enhances the optical and photothermal properties of NPs and thus results in strong scattering and absorption of light. These plasmonic properties enable metal NPs to be used in biodiagnostics, biophysical studies, and medical therapies when they are integrated into biological systems. The strong LSPR scattering displayed by Au NPs when conjugated with specific targeting molecules enables molecule-specific imaging and diagnosis of diseases such as cancer [2-4]. For instance, the cosputtering property of gold-silica nanocomposites opens up the possibility to use these nanocomposites as biosensors in the detection of human ovarian cancer cells [5].

The plasmon resonance properties of metal NPs can be tailored by varying the size, shape, composition, and medium of the NPs, and therefore nanostructures can be designed and geared for specific bioapplications [6] as well as plasmonic-integrated circuits for future electronics and solar cells [7]. The presence of LSPR offers the potential for metal NPs for use in antennas [8]. The synthesis of patterned nanostructures with precise size and spacing present at the surface plays an important role in integrated circuits and surface enhanced Raman scattering (SERS) $[9,10]$. Recent developments in metal NPs include the synthesis of gold-zinc oxide nanocomposite films which are capable of displaying tunable LSPR and Raman signal enhancement for SERS and biosensing [11, 12]. The construction of optically thick 
but physically thin photovoltaic absorbers with thicknesses within the range of tenths to hundredths of nanometres capable of absorbing full solar spectrum has enabled the production of new solar-cell designs with higher efficiency [13]. In addition, large arrays of highly ordered gold nanocups are formed from periodic photoresist templates and used to support surface plasmonic resonance [1].

Various chemical techniques have been implemented to synthesize Au NPs such as citrate reduction of gold salt $[14,15]$, borohydride reduction of gold salt in the presence of a capping agent [16, 17], glycerol reduction of gold salt $[18]$, and other techniques $[19,20]$. Nowadays, noble metals can be processed into monodispersed NPs with controllable compositions and structures using these solution-phase techniques. Despite the advancements of synthesis techniques, the ability of such techniques to synthetically achieve welldispersed metal NPs has been met with limited success. Most metals are present as face-centered cubic structures in the nanometre scale and they tend to nucleate and grow into twinned and multiply twinned particles (MTPs) [21]. Since the intrinsic properties of metal NPs are primarily influenced by their stability, size, and shape, it can be deduced that controlling one of these parameters is the key to fine-tuning the properties of NPs. The seeding growth technique has been shown to be exceptional when it comes to controlling the size of NPs $[22,23]$. In this technique, small metal NPs are first prepared and served as seeds (nucleation sites) which are mixed with a growth solution to synthesize larger particles. The preformed seeds and growth solution are capable of inhibiting secondary or further nucleation, which leads to nonhomogeneous particle size in the stable colloidal system with the help of a stabilizing agent [24].

MMs are composites or artificial electromagnetic materials which consist of resonant unit cells (meta-atoms). The research field of MMs opens a whole new world of fundamental studies. The ability of MMs to display extraordinary electromagnetic properties such as negative refraction index [25, 26], subwavelength imaging [27], and optical cloaking [28] offers benefits in various applications including optical sensing, subdiffraction-limited imaging, nanoscale photolithography, photonic nanocircuits, and a vast range of electronic devices [29]. However, in order for MMs to exhibit unusual electromagnetic responses (negative effective permittivity and permeability values), the meta-atoms must be tailored such that they are substantially smaller than the operating wavelength and the average distance between the neighbouring meta-atoms must be subwavelength in scale [30]. Metal NPs are suitable candidates due to the fact that they possess favourable electronic, optical, and thermal properties. In this study, gold ( $\mathrm{Au}$ ) NPs are chosen due to their established quantum-confinement property and very large specific surface area. However, the main drawback of $\mathrm{Au}$ NPs is that they have a high tendency to aggregate and form agglomerates. It is highly crucial to attain Au NPs with a uniform and smaller size range in order to facilitate and expedite the development of MMs.

In this study, the seeding growth technique is used to synthesize Au NPs and the size, morphology, and stability of the particles are examined using UV-Vis spectrophotometer and zeta potential analyser. These characteristics are then correlated with particle size analysis obtained from transmission electron microscopy. It is generally known that a colloidal system may undergo destabilization such as sedimentation, flocculation, and creaming due to various factors such as particle size, attractive force, and viscosity and it is therefore imperative to examine the destabilization phenomenon of the Au NP colloidal system. Hence, the Turbiscan screening stability tester is used to investigate the stability of the particles for the first time by correlating the destabilization phenomenon with particle migration and particle size variations in the Au NP colloidal system. This is achieved by measuring the light scattering intensities in the Au NPs. A highly stable Au NP colloidal system is efficiently produced in this study, which can be potentially used as a precursor to produce MMs since the size and shape of the particles are well controlled using the seeding growth technique.

\section{Materials and Methods}

2.1. Sample Preparation. Gold (III) chloride hydrate $\left(\mathrm{HAuCl}_{4}\right.$. $\left.x \mathrm{H}_{2} \mathrm{O}\right)$, trisodium citrate $\left(\mathrm{Na}_{3} \mathrm{C}_{6} \mathrm{H}_{5} \mathrm{O}_{7}\right)$, sodium borohydride $\left(\mathrm{NaBH}_{4}\right)$, ascorbic acid $\left(\mathrm{C}_{6} \mathrm{H}_{8} \mathrm{O}_{6}\right)$, and cetyltrimethylammonium bromide (CTAB) were used as received. Ultrapure deionised water (PURELAB Ultra ELGA) was used as the medium during the preparation of all suspensions. The gold seeds and growth solution were prepared independently prior to the seeding growth technique.

A solution consisting of $\mathrm{HAuCl}_{4}\left(2.5 \times 10^{-4} \mathrm{M}\right)$ and $\mathrm{Na}_{3} \mathrm{C}_{6} \mathrm{H}_{5} \mathrm{O}_{7}\left(2.5 \times 10^{-4} \mathrm{M}\right)$ with a volume of $40 \mathrm{~mL}$ was prepared in a conical flask. Following this, $1.2 \mathrm{~mL}$ of freshly prepared $0.1 \mathrm{M} \mathrm{NaBH}$. was added to the solution while stirring and it was observed that the solution immediately turned pink due to the role of the $\mathrm{NaBH}_{4}$, which is a strong reducing agent. The colour transformation of the solution indicates the formation of $\mathrm{Au}$ seeds in the solution (seed solution) with a life span of 2-5 hours. Citrate serves only as a capping agent since it is incapable of reducing gold salt at room temperature.

The following step involves preparation of the stock growth solution, in which $200 \mathrm{~mL}$ of $\mathrm{HAuCl}_{4}\left(2.5 \times 10^{-4} \mathrm{M}\right)$ solution was prepared and mixed with $6 \mathrm{~g}$ of CTAB. The mixture was stirred and heated to obtain a clear orange solution. The solution was then cooled to room temperature and used as the stock growth solution. CTAB was chosen as the surfactant in order to stabilize the particles in the suspension.

A total of four samples were prepared and labelled as I, J, K, and L. Sample I was prepared by mixing $30 \mathrm{~mL}$ of asprepared growth solution with $0.2 \mathrm{~mL}$ of freshly prepared $0.1 \mathrm{M} \mathrm{C}_{6} \mathrm{H}_{8} \mathrm{O}_{6}$, followed by the addition of $10 \mathrm{~mL}$ of seed solution. The solution turned wine red with continuous stirring for 10 minutes. Sample J was prepared according to the previous steps using $36 \mathrm{~mL}$ of as-prepared growth solution, $0.2 \mathrm{~mL}$ of freshly prepared $0.1 \mathrm{M} \mathrm{C}_{6} \mathrm{H}_{8} \mathrm{O}_{6}(\mathrm{AA})$, and $4 \mathrm{~mL}$ of seed solution. The solution was stirred for 10 minutes and its colour turned deep red. Sample K was prepared using the step-by-step particle enlargement method, in which $4 \mathrm{~mL}$ of Sample J (used as the seed solution) was added into 


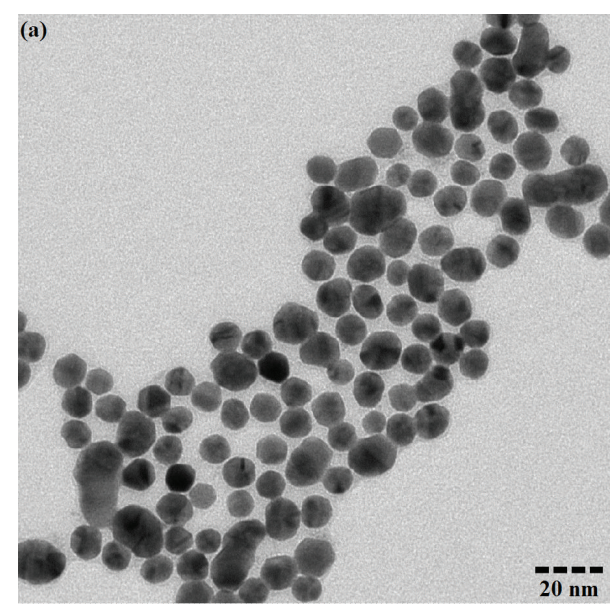

(a)

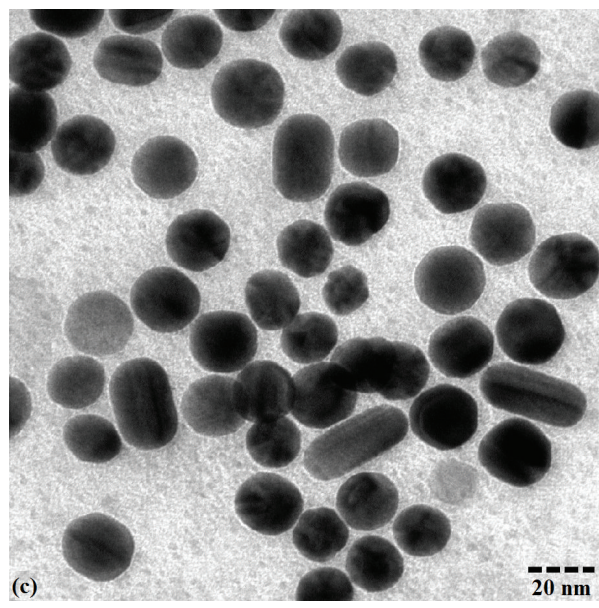

(c)

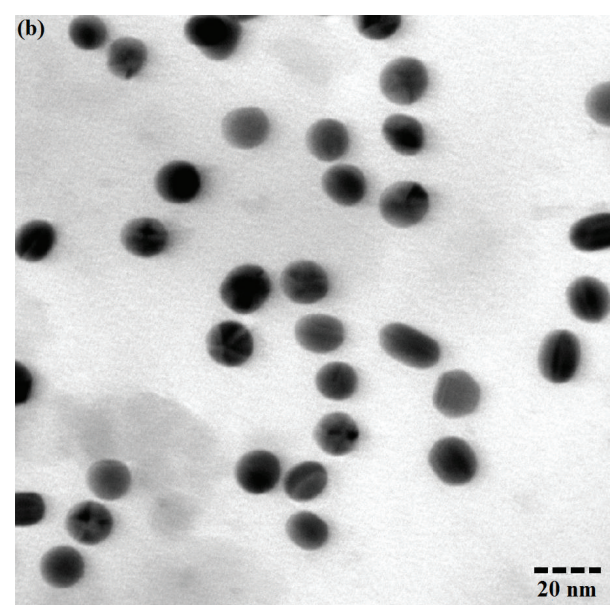

(b)

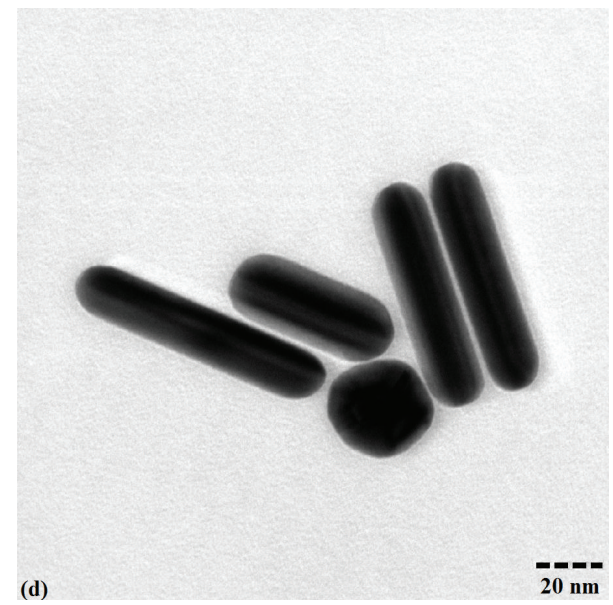

(d)

Figure 1: TEM image of Au NPs (magnification 63000x) for Samples (a) I, (b) J, (c) K, and (d) L.

a mixture containing $36 \mathrm{~mL}$ of as-prepared growth solution and $0.2 \mathrm{~mL}$ of freshly prepared $0.1 \mathrm{M} \mathrm{C}_{6} \mathrm{H}_{8} \mathrm{O}_{6}$. The final solution was stirred vigorously for 10 minutes until its colour turned reddish brown. It shall be noted that the life span of this sample is only 30 minutes after preparation. Sample L was prepared in a similar manner, whereby $4 \mathrm{~mL}$ of solution extracted from Sample K (used as the seed solution) was added into a mixture containing $36 \mathrm{~mL}$ of as-prepared growth solution and $0.2 \mathrm{~mL}$ of freshly prepared $0.1 \mathrm{M} \mathrm{C}_{6} \mathrm{H}_{8} \mathrm{O}_{6}$.

2.2. Characterization. As-synthesised Au suspension was dropped onto a carbon coated copper grid, dried at room temperature, and subsequently examined using a transmission electron microscope (LIBRA 120, Germany) operated at $120 \mathrm{kV}$ in order to examine the morphology and physical structure of the particles.

The absorption spectrum of each sample was recorded using UV-Vis spectrophotometer (Cary Win UV 50, Australia). Plastic disposable cells with an optical path length of $10 \mathrm{~mm}$ and spectral resolution of $1 \mathrm{~nm}$ with a wavelength range of $300-800 \mathrm{~nm}$ were used for spectrophotometric measurements. The optical properties of Au NPs were examined and the variations of particle size were correlated with the results of other characterization techniques.

The Turbiscan Classic MA 2000 screening stability tester (Formulaction Co., France) was used to estimate the dispersion stability of the suspension by monitoring the kinetics of destabilization of the system and correlating them with particle migration and particle size variation. The measurements of the Turbiscan screening stability tester are based on the multiple light scattering method, in which a sample in a standardized bottle is scanned throughout its entire height, which is up to $60 \mathrm{~mm}$ in the backscatter mode at room temperature. This instrument detects the variations of concentration of the dispersed particles in the sample.

Zeta potential measurements were conducted at $25^{\circ} \mathrm{C}$ using Zetasizer Nano ZS (Malvern Instruments Ltd., UK). The zeta potential measurements were recorded by scanning a polycarbonate folded capillary cell (DTS 1061) which contains a sample. It shall be noted that the cell must be rinsed and cleaned prior to the measurements. The sample concentration was fixed at $2.5 \times 10^{-4} \mathrm{M}$ in all cases. 


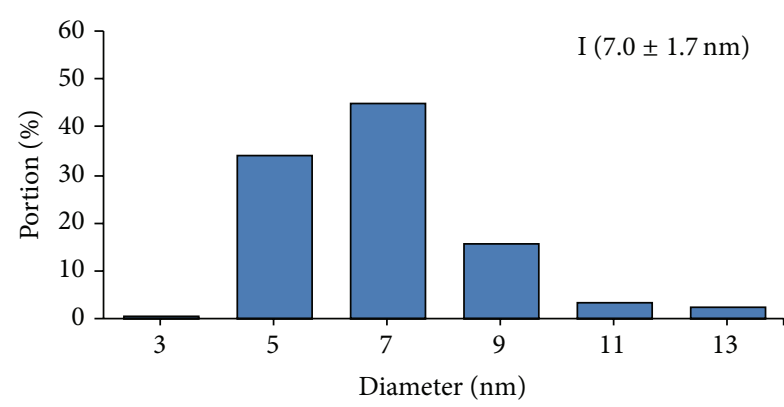

(a)

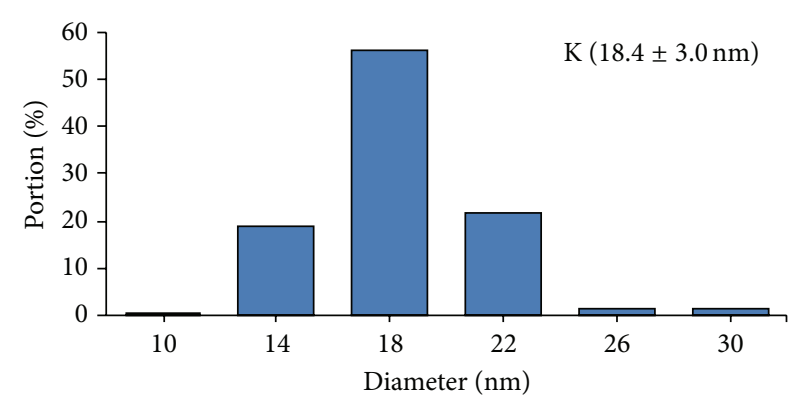

(c)

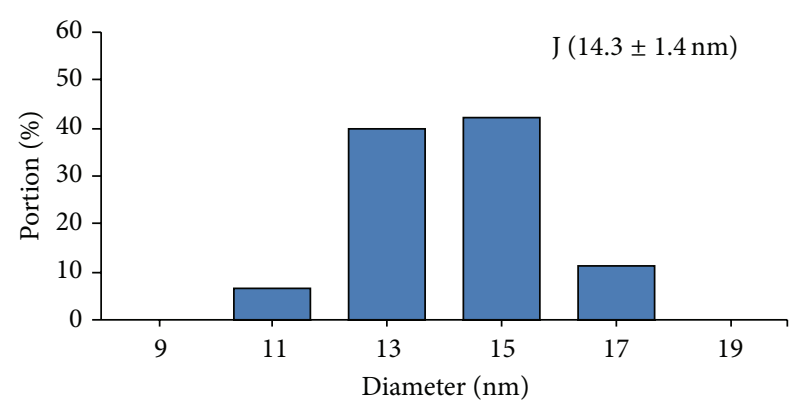

(b)

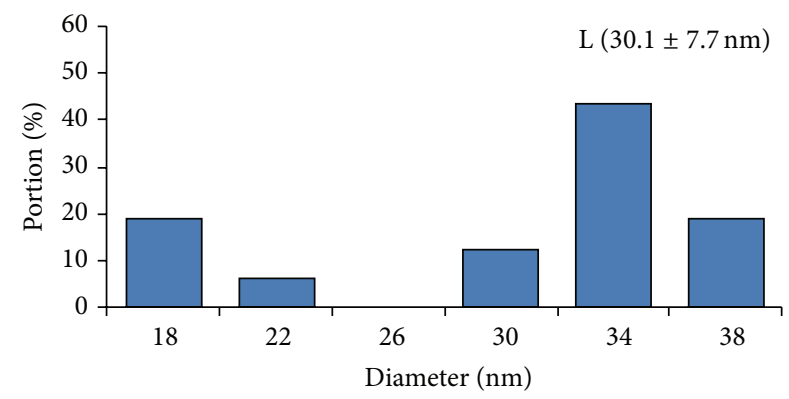

(d)

FIgURE 2: Particle size distributions of Au NPs for all samples.

\section{Results and Discussion}

The TEM images of the synthesized Au NPs for all samples are shown in Figure 1. Sample I (Figure 1(a)) has the smallest particle size $(7.0 \pm 1.7 \mathrm{~nm})$, followed by Sample J $(14.3 \pm 1.4 \mathrm{~nm})$ (Figure 1(b)) and Sample K $(18.4 \pm 3.0 \mathrm{~nm})$ (Figure 1(c)). Sample L has the largest particle size $(30.1 \pm$ $7.7 \mathrm{~nm}$ ) (Figure 1(d)). It can be seen that the particles are nearly spherical with size ranging from 7 to $30 \mathrm{~nm}$. However, rod-shaped particles are present in Sample L, as shown in Figure 1(d). In addition, there are visible spaces between the particles and therefore it can be stated that the particles are well dispersed. Particle agglomeration is not observed in these images, which implies that the Au NPs are homogeneously dispersed when deionised water is used as the medium in the synthesis. The samples also exhibit good stability. In the seeding growth technique, the seeds (Au NPs) are first produced and added to a growth solution which contains more $\mathrm{Au}$ ions as well as CTAB surfactant which will induce anisotropic growth of NPs. The seeds become tremendously small $(1-5 \mathrm{~nm})$, as evidenced by the TEM as well. The seeds have narrow size distribution when the strong reducing agent $\left(\mathrm{NaBH}_{4}\right)$ reduces the gold salt $\left(\mathrm{Au}^{3+}\right)$ to gold $(\mathrm{Au})$ under high chemical supersaturation followed by capping with citrate, which increases the nucleation rate. Meanwhile, the growth solution contains a weaker reducing agent (AA) which reduces the gold salt to an intermediate state (Au ions). This enables only catalysed reduction at the surface of the Au NPs. The suitability of growth solution is essential in controlling the final particle size. However, the presence of seeds often induces further nucleation rather than growth, resulting in a broad particle size distribution. Hence, a step-by-step particle enlargement method is performed to inhibit further nucleation, resulting in the formation of particles with homogeneous size. The CTAB medium provides a growth environment which stabilises the Au NPs formed at the final stage by inhibiting particle aggregation during growth $[20,31,32]$.

The particle size distributions for Samples I, J, K, and $\mathrm{L}$ are presented in Figure 2. It can be observed that the particles in Sample J have the highest sphericity compared to the other samples. Sample J has a size distribution with the smallest standard deviation (1.4 nm), as shown in Figure 2(b). In contrast, Sample $\mathrm{L}$ has a size distribution with the largest standard deviation $(7.7 \mathrm{~nm})$, as shown in Figure $2(\mathrm{~d})$. The particles in Sample L are spherical and rod-shaped, which is attributed to the larger particles in Sample K used as the seeds in the preparation of Sample L. The morphology and dimension of $\mathrm{Au}$ NPs are solely dependent on the nature of the seed particles $[19,23]$ since the concentrations of the reducing agents $\left(\mathrm{NaBH}_{4}\right.$ and $\left.\mathrm{AA}\right)$, capping agent (citrate), surfactant (CTAB), and reactant (Au ions) are constant. In addition, it is believed that the growth process is due to kinetic conditions. It has been shown in previous studies that counter ions and additives play a vital role in directing the growth and final shape of the NPs. Therefore, the particle shape is likely influenced by the interplay between the growth kinetics of the particles and the faceting tendency of the stabilizing agents (citrate and CTAB), which facilitate the spherical shape of Au NPs. Meanwhile, the rod-like particles are found in relatively less numbers in the overall Sample L. The CTAB transforms into cationic surfactant micelles, which serve as a "soft template" in directing the nanoparticle growth and provide colloidal stability for the synthesized Au NPs 


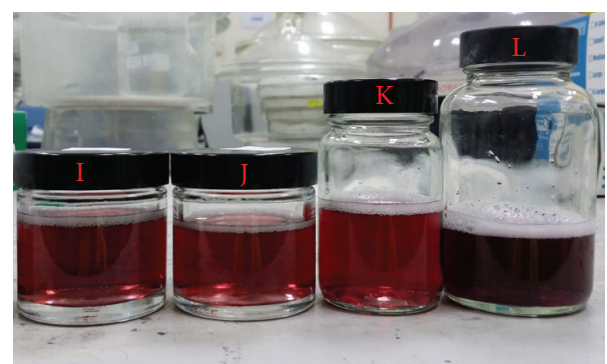

(a)

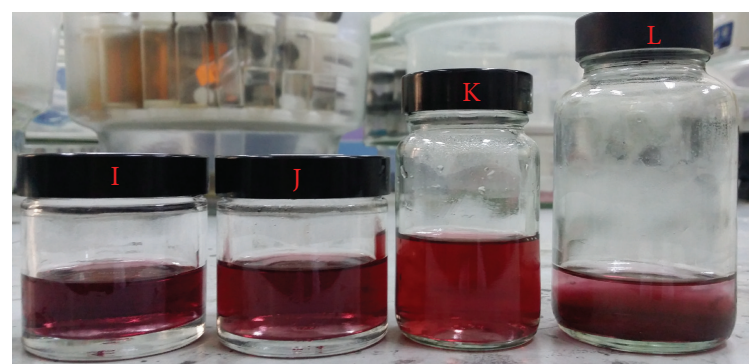

(b)

FIGURE 3: Sedimentation behaviour of Au suspension for all samples: (a) as-synthesized and (b) after 2 months.

$[23,31,32]$. This results in size polydispersity, which in turn gives a broader size distribution.

The stability of Samples I, J, K, and L was inspected visually by monitoring the sedimentation behaviour of the Au suspension, and the results are shown in Figure 3. It can be seen that all samples remain the same after 2 months with the exception of Sample L. The surface charge modification of Au NPs caused by the CTAB surfactant creates a repulsive force between the particles, which stabilizes the NPs [23]. However, the large particles in Sample L eventually settle down at the bottom of the jar due to their weight. The weight of the particles in Sample L overcomes the repulsive force between the particles and promotes sedimentation. The invariant changes in colour for all samples also indicate that there are no changes in particle size over an extended period, which results in a homogeneous particle size.

The optical absorption spectra of all samples within a wavelength range of $300-800 \mathrm{~nm}$ are shown in Figure 4. It can be seen that all samples exhibit a single intense peak within a range of $520-535 \mathrm{~nm}$, which is assigned to the surface plasmon resonance (SPR) of Au NPs [18, 33, 34]. The appearance of SPR peaks indicates that the Au NPs have been synthesized successfully using the seeding growth technique. The SPR peak shifts gradually towards a longer wavelength (redshift), which agrees well with the increase in particle size shown in Figures 1 and 2. Sample L exhibits the most apparent redshift in the SPR peak along the parallel direction relative to Sample K, with a value of $\approx 8 \mathrm{~nm}$. This pronounced redshift indicates the largest Au NPs found in Sample L [34].

The backscattered light profiles of Au NPs over the total height of the sample dispersed in deionised water are shown in Figure 5, in which measurements are taken over a period of 5 days. The backscattered light flux measured in percent (\%) varies as a function of the density of suspension within the medium. In general, different concentrations of dispersed particles at the top and bottom segments of each sample will result in different backscattered light signals. Each sample was scanned in a standardised tube with a sample height of $50 \mathrm{~mm}$, in which the incident light has a wavelength of $850 \mathrm{~nm}$. The samples exhibit similar patterns with regard to their backscatter profiles, whereby the backscattered light signals at the top segment of the profiles $(40-45 \mathrm{~mm})$ decrease with time. However, the backscattered light signals at the bottom segment of the profiles $(1-5 \mathrm{~mm})$ increase with time,

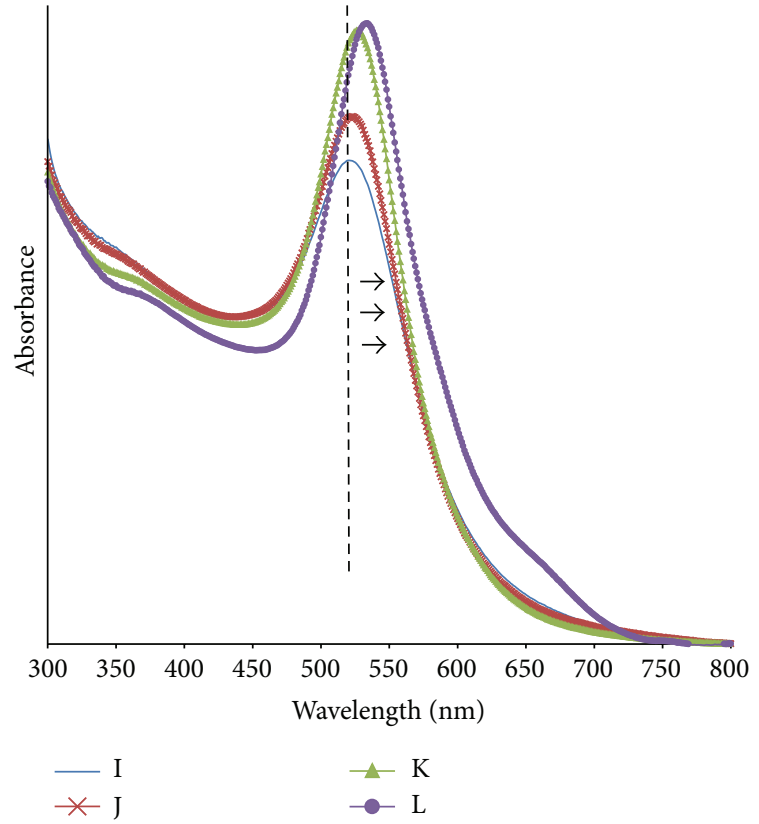

FIGURE 4: Optical absorption spectra of Au NPs for all samples.

though the signals are rather indistinct. This observation is attributed to a decrease in the concentration of the dispersed particles within the top region of the samples, which is due to particle sedimentation $[35,36]$. It is observed that the Au NPs begin to sediment slightly after about 5 days.

The peak thickness kinetics (absolute or phase thickness) versus time was computed based on the top segment of the profiles $(40-45 \mathrm{~mm})$ in order to investigate the particle migration phenomenon, and the plots are shown in Figure 6. In general, a higher gradient indicates an increase in the sedimentation rate of the particles in the suspension [37]. It can be deduced from the plots that the sedimentation of particles occurs over a longer period since the gradients are not steep, in which the change in peak thickness kinetics (readings in the $y$-axis) with respect to time is negligibly small. The sedimentation phenomenon is rather minor, which indicates that the Au NPs are highly stable. The NPs are stabilized effectively and sedimentation is prevented due to the CTAB surfactant [23]. However, the sample with 


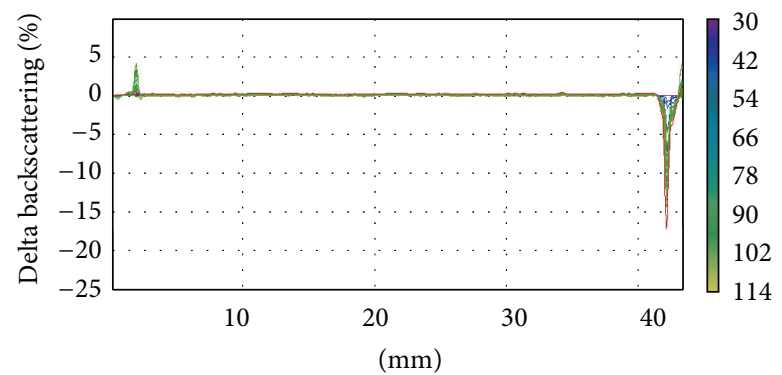

K

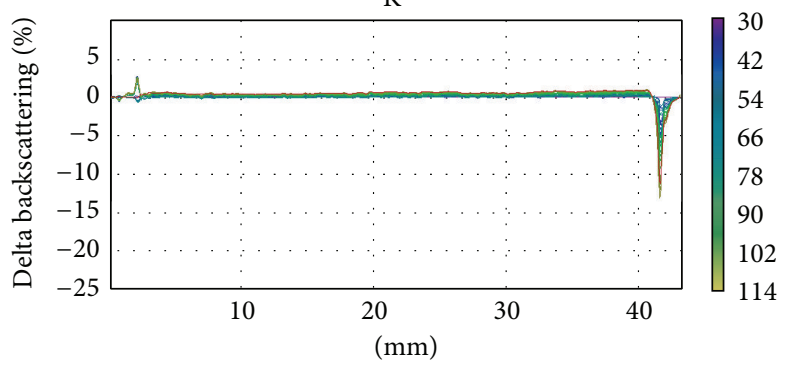

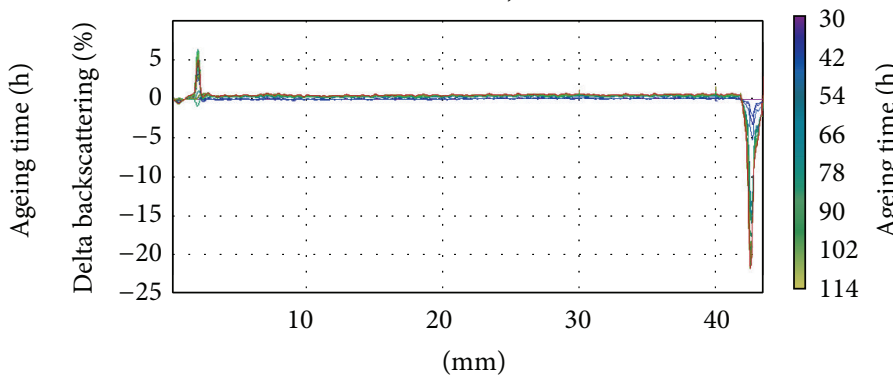

$\mathrm{L}$

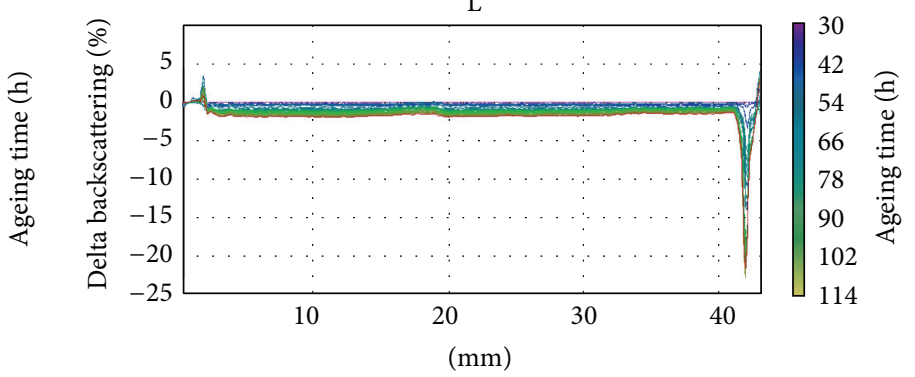

FIGURE 5: Backscattered light profiles of Au NPs for all samples with different particle size.

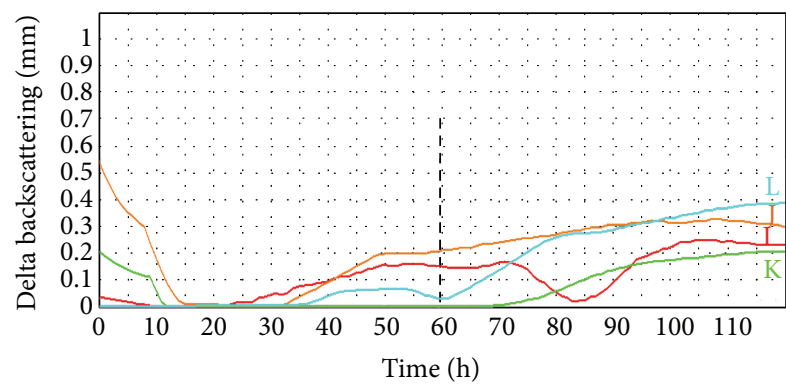

FIgURE 6: Peak thickness kinetics of Au NPs at the top segment for all samples.

the largest mean particle size (Sample L) has the highest gradient after 60 hours, which indicates that it has the highest sedimentation rate among all samples. The large particles settle down eventually at the bottom of the jar for Sample L due to their weight, as evidenced from Figure 3.

The middle segment of each profile $(5-40 \mathrm{~mm})$ is nearly flat, indicating that the particle size for each sample remains constant, as shown in Figure 5. However, the backscatter intensity over the total height for Sample L (sample with the largest particle size) slightly decreases with time which is barely noticeable but should not be ignored, as this implies that the size of the Au NPs increases over time. The increase in particle size is obvious when the mean value kinetics of Sample L is computed and plotted based on the middle segment of the profile $(5-40 \mathrm{~mm})$, in which the change is homogeneous, as shown in Figure 7. The mean value kinetics is used to explain the variation of particle size [36]. In this case, the mean kinetic value of Sample L slightly decreases with time, whereas the other samples exhibit an almost

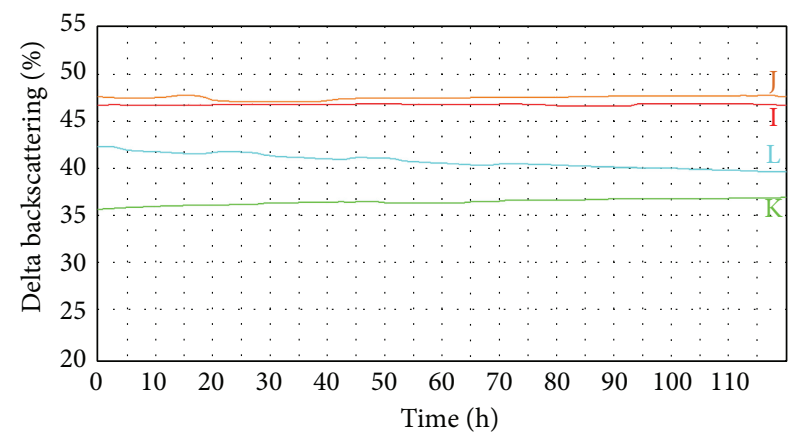

Figure 7: Mean value kinetics of Au NPs at the middle segment for all samples.

TABLE 1: Mean particle size, surface plasmon resonance, and zeta potential value of $\mathrm{Au}$ nanoparticles for all samples.

\begin{tabular}{lcccc}
\hline Sample & $\begin{array}{c}\text { Mean particle } \\
\text { Size }(\mathrm{nm})\end{array}$ & $\begin{array}{c}\text { Surface plasmon } \\
\text { Resonance }(\mathrm{nm})\end{array}$ & $\begin{array}{c}\text { Zeta potential }(\mathrm{mV}) \\
\text { As-synthesized }\end{array}$ & 2 months \\
\hline $\mathrm{I}$ & $7.0 \pm 1.7$ & 520 & 80.3 & 58.7 \\
$\mathrm{~J}$ & $14.3 \pm 1.4$ & 523 & 78.6 & 40.9 \\
$\mathrm{~K}$ & $18.4 \pm 3.0$ & 527 & 73.2 & 42.4 \\
$\mathrm{~L}$ & $30.1 \pm 7.7$ & 534 & 60.3 & 38.9 \\
\hline
\end{tabular}

constant trend. The small decrement in the mean value kinetics implies an increase in particle size, which is probably due to a slight agglomeration of the particles. However, the particles in Sample L are still well dispersed in the deionised water.

The results of the TEM, optical absorption spectra, and zeta potential measurements are summarized in Table 1. It is 
apparent that an increase in particle size corresponds to an increase in SPR wavelength for all samples, indicating that the samples are red-shifted. These data are in good agreement with the zeta potential values. It is widely accepted that a higher zeta potential value indicates an improvement in the stability of the suspension [35] due to the greater repulsive force formed between the Au NPs which keeps the particles apart from one another. In general, the larger the mean particle size of Au NPs, the lower the stability of the NPs. The repulsive force decreases with an increase in particle size due to a decrease in surface contact area which reduces the zeta potential value [20]. The results of the particle size agree well with the zeta potential values, whereby Sample L has the largest mean particle size but the lowest zeta potential value. It is for this reason that Sample $\mathrm{L}$ exhibits the highest sedimentation rate among all samples, as evidenced from Figures 3 and 6.

Obviously, a larger mean particle size indicates an increase in weight of the Au NPs, which promotes particle sedimentation. In addition, the number of atoms certainly will increase the weight of the particles which is reflected by the growth rate as well. The weight of the Au NPs overcomes the repulsive force between the individual particles which is associated with the zeta potential values and thus the stability of Au NPs in the suspension decreases with time gradually. However, it is found that all samples still exhibit a considerable zeta potential value after 2 months, which indicates that the suspensions are highly stable.

\section{Conclusions}

In this study, a stable gold nanoparticle (Au NP) colloidal system has been prepared successfully using a seeding growth technique coupled with a step-by-step particle enlargement route. It is found that the size of the spherical Au NPs is within a range of 7-30 $\mathrm{nm}$ and the particles are well dispersed in deionised water. However, it is found that rod-shaped particles are present in the sample with the largest mean particle size $(30 \mathrm{~nm})$, that is, Sample L. The stability of the Au NPs has been investigated experimentally using the Turbiscan screening stability tester and zeta potential analyser. The backscattered light profiles imply that the Au NP suspensions are stable while the peak thickness kinetics reveals that the particle sedimentation is rather minor. The mean value kinetics indicates that there is negligible particle agglomeration in the suspensions over a period of 5 days. The zeta potential values reflect the repulsive force between particles and hence the stability of the suspensions. It is found that the mean particle size of the Au NPs is strongly correlated with the zeta potential value, in which larger particles decrease the surface contact area and stability of the suspension governed by the zeta potential. The increase in particle size increases the weight of the Au NPs which eventually overcomes the repulsive force between the individual particles. Thus, the sample with the largest mean particle size (Sample L) has the highest sedimentation rate compared to other samples. The samples are found to remain stable over a period of 2 months and are therefore suitable for the production of MMs, with the exception of Sample L.

\section{Conflict of Interests}

The authors declare that there is no conflict of interests regarding the publication of this paper.

\section{Acknowledgments}

The authors graciously acknowledge the financial support provided by the University of Malaya under the UMRG Research Grant (RP011C-13AET) and Postgraduate Research Grant (PPP) (Grant no. PG117-2013A). The authors also wish to express their appreciation to Prof. Dr. Rauzah Hashim from Department of Chemistry, University of Malaya, for offering guidance and technical support regarding zeta potential measurements using Zetasizer Nano ZS.

\section{References}

[1] H. G. Svavarsson, J. W. Yoon, M. Shokooh-Saremi, S. H. Song, and R. Magnusson, "Fabrication and characterization of large, perfectly periodic arrays of metallic nanocups," Plasmonics, vol. 7, no. 4, pp. 653-657, 2012.

[2] P. Alivisatos, "The use of nanocrystals in biological detection," Nature Biotechnology, vol. 22, no. 1, pp. 47-52, 2004.

[3] N. L. Rosi and C. A. Mirkin, "Nanostructures in biodiagnostics," Chemical Reviews, vol. 105, no. 4, pp. 1547-1562, 2005.

[4] B. M. Reinhard, S. Sheikholeslami, A. Mastroianni, A. P. Alivisatos, and J. Liphardt, "Use of plasmon coupling to reveal the dynamics of DNA bending and cleavage by single EcoRV restriction enzymes," Proceedings of the National Academy of Sciences of the United States of America, vol. 104, no. 8, pp. 26672672, 2007.

[5] Y. K. Mishra, S. Mohapatra, D. K. Avasthi et al., "Gold-silica nanocomposites for the detection of human ovarian cancer cells: a preliminary study," Nanotechnology, vol. 18, no. 34, Article ID 345606, 2007.

[6] P. K. Jain, X. Huang, I. H. El-Sayed, and M. A. El-Sayed, "Noble metals on the nanoscale: optical and photothermal properties and some applications in imaging, sensing, biology, and medicine," Accounts of Chemical Research, vol. 41, no. 12, pp. 1578-1586, 2008.

[7] S. Roh, T. Chung, and B. Lee, "Overview of the characteristics of micro- and nano-structured surface plasmon resonance sensors," Sensors, vol. 11, no. 2, pp. 1565-1588, 2011.

[8] H.-M. Gong, L. Zhou, X.-R. Su, S. Xiao, S.-D. Liu, and Q.-Q. Wang, "Illuminating dark plasmons of silver nanoantenna rings to enhance exciton-plasmon interactions," Advanced Functional Materials, vol. 19, no. 2, pp. 298-303, 2009.

[9] L. Fei, D. W. Brandl, Y. A. Urzhumov et al., "Metallic nanoparticle arrays: a common substrate for both surface-enhanced raman scattering and surface-enhanced infrared absorption," ACS Nano, vol. 2, no. 4, pp. 707-718, 2008.

[10] M. Rycenga, C. M. Cobley, J. Zeng et al., "Controlling the synthesis and assembly of silver nanostructures for plasmonic applications," Chemical Reviews, vol. 111, no. 6, pp. 3669-3712, 2011.

[11] Y. K. Mishra, S. Mohapatra, R. Singhal, D. K. Avasthi, D. C. Agarwal, and S. B. Ogale, "Au-ZnO: a tunable localized surface plasmonic nanocomposite," Applied Physics Letters, vol. 92, no. 4, Article ID 043107, 2008. 
[12] Y. K. Mishra, V. S. K. Chakravadhanula, V. Hrkac et al., "Crystal growth behaviour in $\mathrm{Au}-\mathrm{ZnO}$ nanocomposite under different annealing environments and photoswitchability," Journal of Applied Physics, vol. 112, no. 6, Article ID 064308, 2012.

[13] H. A. Atwater and A. Polman, "Plasmonics for improved photovoltaic devices," Nature Materials, vol. 9, no. 3, pp. 205-213, 2010.

[14] J. Turkevich, G. Garton, and P. C. Stevenson, "The color of colloidal gold," Journal of Colloid Science, vol. 9, no. 1, pp. 2635, 1954.

[15] G. Frens, "Controlled nucleation for the regulation of the particle size in monodisperse gold suspensions," Nature, vol. 241, no. 105, pp. 20-22, 1973.

[16] M. Brust, M. Walker, D. Bethell, D. J. Schiffrin, and R. Whyman, "Synthesis of thiol-derivatised gold nanoparticles in a twophase liquid-liquid system," Chemical Communications, no. 7, pp. 801-802, 1994.

[17] M. J. Hostetler, J. E. Wingate, C.-J. Zhong et al., "Alkanethiolate gold cluster molecules with core diameters from 1.5 to $5.2 \mathrm{~nm}$ : core and monolayer properties as a function of core size," Langmuir, vol. 14, no. 1, pp. 17-30, 1998.

[18] P. Nalawade, T. Mukherjee, and S. Kapoor, "Green synthesis of gold nanoparticles using glycerol as a reducing agent," Advances in Nanoparticles, vol. 2, no. 2, pp. 78-86, 2013.

[19] T. K. Sau and C. J. Murphy, "Room temperature, high-yield synthesis of multiple shapes of gold nanoparticles in aqueous solution," Journal of the American Chemical Society, vol. 126, no. 28, pp. 8648-8649, 2004.

[20] L. H. Bac, J. S. Kim, and J. C. Kim, "Size, optical and stability properties of gold nanoparticles synthesized by electrical explosion of wire in different aqueous media," Reviews on Advanced Materials Science, vol. 28, no. 2, pp. 117-121, 2011.

[21] J. G. Allpress and J. V. Sanders, "The structure and orientation of crystals in deposits of metals on mica," Surface Science, vol. 7, no. 1, pp. 1-25, 1967.

[22] K. R. Brown, D. G. Walter, and M. J. Natan, "Seeding of colloidal Au nanoparticle solutions. 2. Improved control of particle size \& shape," Chemistry of Materials, vol. 12, no. 2, pp. 306-313, 2000.

[23] N. R. Jana, L. Gearheart, and C. J. Murphy, "Seeding growth for size control of 5-40 nm diameter gold nanoparticles," Langmuir, vol. 17, no. 22, pp. 6782-6786, 2001.

[24] N. R. Jana, L. Gearheart, and C. J. Murphy, "Evidence for seedmediated nucleation in the chemical reduction of gold salts to gold nanoparticles," Chemistry of Materials, vol. 13, no. 7, pp. 2313-2322, 2001.

[25] W. J. Padilla, D. N. Basov, and D. R. Smith, "Negative refractive index metamaterials," Materials Today, vol. 9, no. 7-8, pp. 28-35, 2006.

[26] X.-Y. Jiang, Ed., Metamaterial, InTech, Rijeka, Croatia, 2012.

[27] Z. Liu, H. Lee, Y. Xiong, C. Sun, and X. Zhang, "Far-field optical hyperlens magnifying sub-diffraction-limited objects," Science, vol. 315, no. 5819, p. 1686, 2007.

[28] A. Alù and N. Engheta, "Multifrequency optical invisibility cloak with layered plasmonic shells," Physical Review Letters, vol. 100, no. 11, Article ID 113901, 2008.

[29] W. Cai and V. Shalaev, Optical Mematerials: Fundamentals and Applications, Springer, New York, NY, USA, 2010.

[30] S. Mühlig, C. Rockstuhl, V. Yannopapas, T. Bürgi, N. Shalkevich, and F. Lederer, "Optical properties of a fabricated selfassembled bottom-up bulk metamaterial," Optics Express, vol. 19, no. 10, pp. 9607-9616, 2011.
[31] S. Eustis and M. A. El-Sayed, "Why gold nanoparticles are more precious than pretty gold: noble metal surface plasmon resonance and its enhancement of the radiative and nonradiative properties of nanocrystals of different shapes," Chemical Society Reviews, vol. 35, no. 3, pp. 209-217, 2006.

[32] M. Grzelczak, J. Pérez-Juste, P. Mulvaney, and L. M. Liz-Marzán, "Shape control in gold nanoparticle synthesis," Chemical Society Reviews, vol. 37, no. 9, pp. 1783-1791, 2008.

[33] A. Mishra, P. Tripathy, S. Ram, and H.-J. Fecht, "Optical properties in nanofluids of gold nanoparticles in poly(vinylpyrrolidone)," Journal of Nanoscience and Nanotechnology, vol. 9, no. 7, pp. 4342-4347, 2009.

[34] P. K. Jain, K. S. Lee, I. H. El-Sayed, and M. A. El-Sayed, "Calculated absorption and scattering properties of gold nanoparticles of different size, shape, and composition: applications in biological imaging and biomedicine," Journal of Physical Chemistry B, vol. 110, no. 14, pp. 7238-7248, 2006.

[35] O. Mengual, G. Meunier, I. Cayré, K. Puech, and P. Snabre, "Characterisation of instability of concentrated dispersions by a new optical analyser: the TURBISCAN MA 1000," Colloids and Surfaces A: Physicochemical and Engineering Aspects, vol. 152, no. 1-2, pp. 111-123, 1999.

[36] H. Buron, O. Mengual, G. Meunier, I. Cayré, and P. Snabre, "Optical characterization of concentrated dispersions: applications to laboratory analyses and on-line process monitoring and control," Polymer International, vol. 53, no. 9, pp. 1205-1209, 2004.

[37] N. Azema, "Sedimentation behaviour study by three optical methods-granulometric and electrophoresis measurements, dispersion optical analyser," Powder Technology, vol. 165, no. 3, pp. 133-139, 2006. 

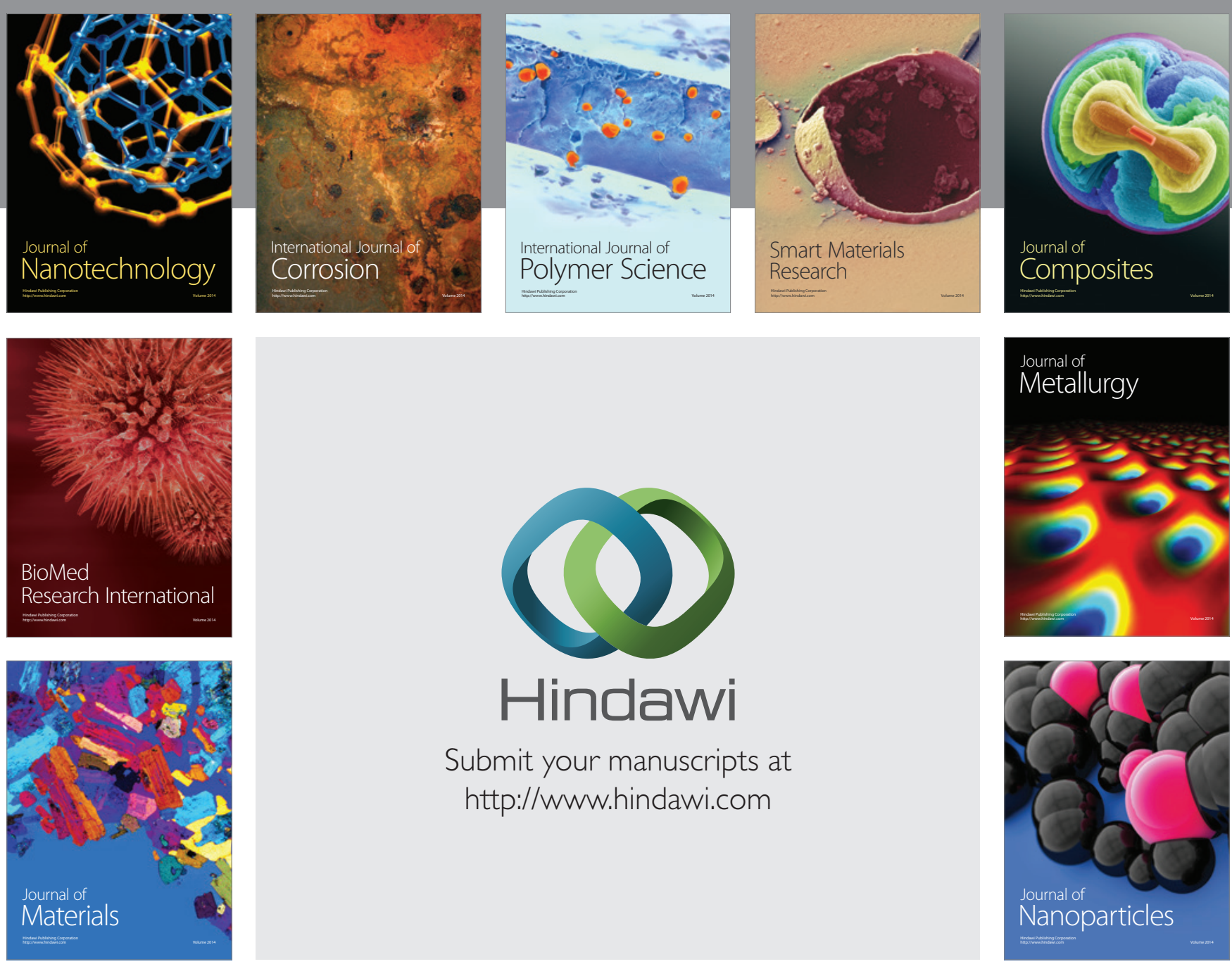

Submit your manuscripts at http://www.hindawi.com
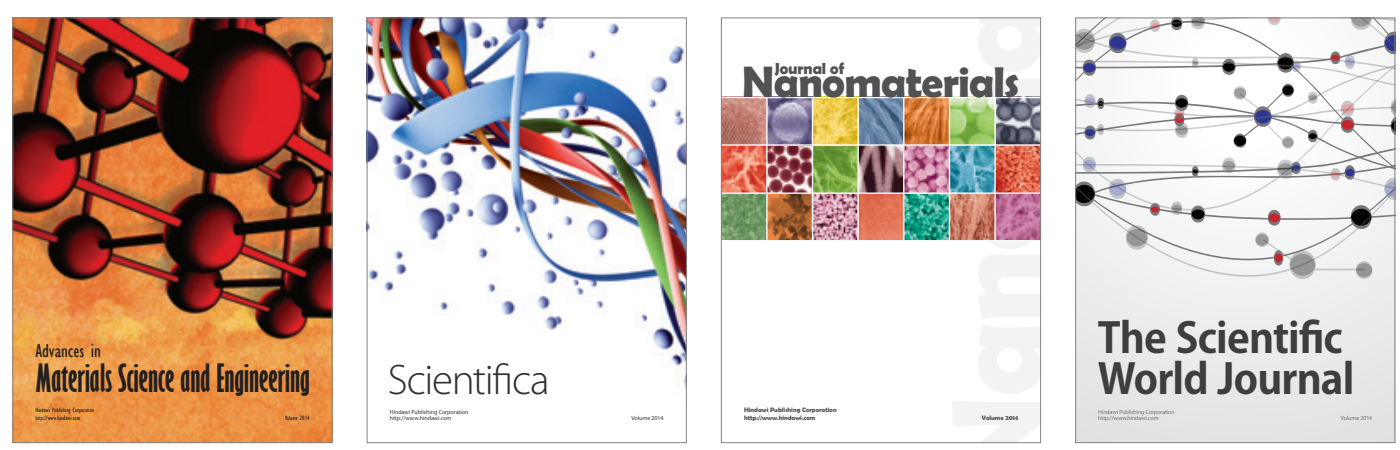

\section{The Scientific World Journal}
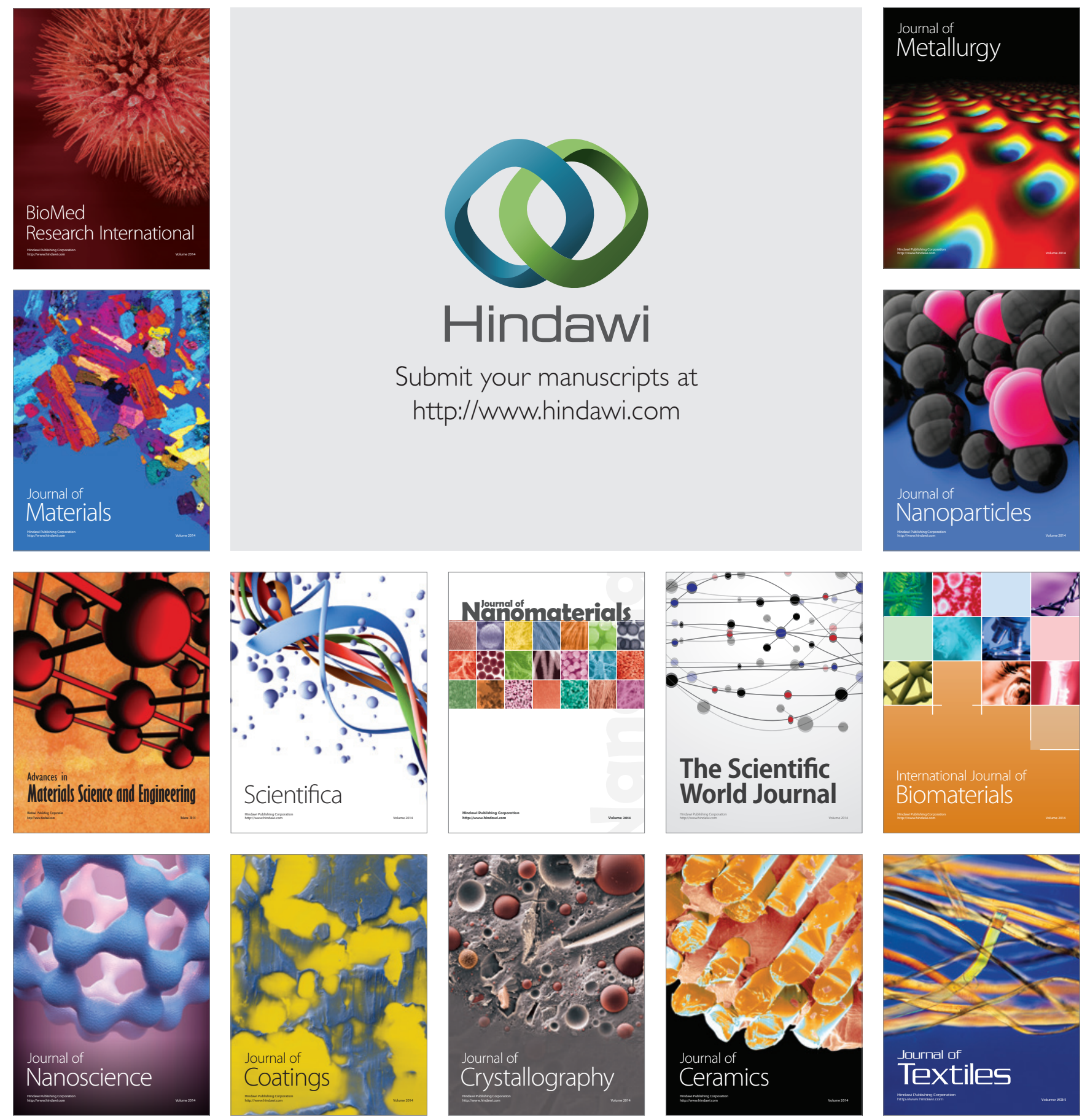